\title{
LncRNA KCNQ10T1 promoted hepatitis C virus-induced pyroptosis of $\beta$-cell through mediating the miR-223-3p/NLRP3 axis
}

\author{
Ben Niu ${ }^{1}$, Lixuan Yao ${ }^{1}$, Yating Zhang ${ }^{1}$, Xueshan Xia ${ }^{2}$, Heng Su ${ }^{1}$ \\ ${ }^{1}$ Department of Endocrinology and Metabolism, The First People's Hospital of Yunnan Province, The Affiliated Hospital of Kunming University \\ of Science and Technology, Kunming, China; ${ }^{2}$ Faculty of Life Science and Technology, Kunming University of Science and Technology, Kunming, \\ China \\ Contributions: (I) Conception and design: B Niu, X Xia; (II) Administrative support: X Xia; (III) Provision of study materials or patients: H Su; (IV) \\ Collection and assembly of data: B Niu, L Yao; (V) Data analysis and interpretation: Y Zhang; (VI) Manuscript writing: All authors; (VII) Final \\ approval of manuscript: All authors. \\ Correspondence to: Heng Su. Department of Endocrinology and Metabolism, The First People's Hospital of Yunnan Province, The Affiliated Hospital \\ of Kunming University of Science and Technology, No. 157 Jinbi Road, Kunming 650032, China. Email: suhengynsdyrmyy@163.com.
}

Background: Type 2 diabetes is a well described extra-hepatic manifestation of hepatitis C virus (HCV) infection. This study aimed to explore the potential mechanism of KCNQ1 overlapping transcript 1 (KCNQ1OT1) in type 2 diabetes mellitus (T2DM) caused by HCV infection.

Methods: Min6 cells were infected with HCV to establish a vitro model, and the HCV copy number was detected by real-time quantitative PCR (RT-qPCR). The mRNA and protein expressions of IL-1 $\beta$, IL18, NLRP3, caspase-1, and GSDMD were analyzed by RT-qPCR and Western blot. Flow cytometry and TUNEL assay were used to evaluate the pyroptosis of cells and enzyme-linked immunosorbent assay (ELISA) detected the secretion of insulin. A dual luciferase reporter gene assay then verified the targeting relationship of KCNQ1OT1, miRNA-223-3p, and NLRP3.

Results: KCNQ1OT1 was highly expressed in HCV-infected T2DM patients and HCV-infected $\beta$-cells. Silencing KCNQ1OT1 inhibited $\beta$-cell pyroptosis by regulating miR-223-3p/NLRP3, and inhibition of miR-223-3p or overexpression of NLRP3 reversed the pyroptosis by silencing KCNQ1OT1.

Conclusions: Our findings indicate KCNQ1OT1 promotes HCV-infected $\beta$-cell pyroptosis through the miRNA-223-3p/NLRP3 axis, effecting the production of insulin and accelerating the occurrence and development of T2DM.Regulating KCNQ1OT1 and its target genes will help to better understand the pathogenesis of T2DM induced by HCV infection and provide new theoretical foundations and therapeutic targets.

Keywords: $\beta$-cell; hepatitis C virus (HCV); KCNQ1 overlapping transcript 1 (KCNQ1OT1); miR-223-3p; pyroptosis

Submitted Jul 01, 2021. Accepted for publication Aug 12, 2021.

doi: 10.21037/atm-21-3862

View this article at: https://dx.doi.org/10.21037/atm-21-3862

\section{Introduction}

Type 2 diabetes mellitus (T2DM) is a metabolic syndrome caused by insufficient insulin secretion and insulin resistance. The disease affects the metabolism of carbohydrates, fats, and proteins, causing hyperglycemia and related complications and disease (1-3). The inflammatory environment aggravates $\beta$-cell damage and promotes the occurrence of T2DM. Recent studies have shown that patients with hepatitis $\mathrm{C}$ virus $(\mathrm{HCV})$ have a higher risk of developing T2DM than the general population (4). T2DM has also been confirmed as an extrahepatic manifestation of $\mathrm{HCV}$ infection, which affects insulin signal transduction cascade $(5,6)$. In addition, the activation of proinflammatory mediators caused by chronic infection can 
interfere with insulin signal transduction, thereby reducing the sensitivity to insulin and leading to liver and peripheral insulin resistance $(7,8)$. However, the interaction between HCV infection and T2DM remains unexplained, and it is necessary to clarify the potential mechanism of T2DM caused by HCV infection.

Many epidemiological studies have shown an association between T2DM and HCV, involving direct viral effects, insulin resistance, proinflammatory cytokines, chemokines, suppressors of cytokine signaling, and other immunemediated mechanisms (9). Inflammation is one of importantly related to the pathogenesis of diabetes and $\mathrm{HCV}$. The apoptosis and pyroptosis are the main cause of $\beta$-cell death. Apoptosis is a very tightly programmed cell death with distinct biochemical and genetic pathways (10). Pyroptosis is a kind of programmed cell necrosis characterized by cells continuing to swell until the cell membrane ruptures, leading to the uncontrolled release of cell inflammatory content and activating a strong inflammatory response $(11,12)$. In recent years, studies have found that cell pyroptosis is related to diabetes, and $\beta$-cell pyroptosis is a new hot spot in the study of new pathogenesis related to T2DM $(13,14)$, suggesting that inflammation may promote the occurrence and development of T2DM by pyroptosis. Additionally, HCV infection can cause pyroptosis of infected liver cancer cells and surrounding non-infected cells affecting the occurrence and development of cancer $(15,16)$ and $\mathrm{HCV}$ induce the occurrence of T2DM (17). However, how the HCV infection in $\beta$-cell pyroptosis remains elusive.

Long non-coding RNA (lncRNA) is a heterogeneous non-coding RNA with a length of more than 200 nucleotides (18-20) and is involved in regulating the development of liver cancer caused by HCV infection $(21,22)$. In addition, some studies have reported that lncRNA regulated $\beta$-cells to affect insulin synthesis and secretion (23-25). While it has been reported that lncRNA KCNQ1 overlapping transcript 1 (KCNQ1OT1) is related to many diseases and plays an important role in diabetes-related complications (26), the function of KCNQ1OT1 in HCV infection on diabetes is still unknown. The common mechanism by which lncRNA works is to act as a competitive endogenous RNA (ceRNA), scaffold, interact with proteins, or form membraneless organelles. lncRNA can bind to the complementary binding sites of microRNAs (miRNAs), which regulate the expression of genes by covering the 3'-UTR of downstream target genes through sponges. miRNAs regulate $30 \%$ of proteincoding genes in the human body and have become a research hotspot in the field of life sciences in recent years (27-29). miR-223-3p is a member of the miRNA family, not only as novel non-invasive markers for the early detection of $\mathrm{HCV}$ positive cirrhosis and hepatocellular carcinoma (HCC), but also as a new screening biomarker to identify subjects with prediabetes at high risk of developing diabetes $(30,31)$. A recent study showed that knockdown of KCNQ1OT1 ameliorates cardiomyocytes pyroptosis by regulating miR214-3p/caspase- 1 axis in diabetic mice (26). In this study, we explored the expression of KCNQ1OT1 in HCV-infected T2DM patients and $\beta$-cells, as well as the mechanism of KCNQ1OT1 regulates $\mathrm{HCV}$-induced pyroptosis of $\beta$-cells by targeting miRNA-223-3p/NLRP3 axis. We found that KCNQ1OT1 was highly expressed in HCVinfected T2DM patients and $\beta$-cells, and miRNA-223-3p showed low expression in $\mathrm{HCV}$-infected $\beta$-cells. At the molecular level, we verified that KCNQ1OT1 regulates $\mathrm{HCV}$-induced pyroptosis of $\beta$-cells and inhibits the production of insulin through the miRNA-223-3p/NLRP3 axis. We present the following article in accordance with the MDAR reporting checklist (available at https://dx.doi. org/10.21037/atm-21-3862).

\section{Methods}

\section{Patients statement}

Serum samples from twenty patients with $\mathrm{HCV}$-infected T2DM and twenty non-HCV-infected T2DM patients were obtained from the First People's Hospital of Yunnan Province. None of the patients had coronary artery disease, hypertension, or other heart diseases. The study received approval from the ethics committee of the First People's Hospital of Yunnan Province (KHLL2020-KY059), and all participants signed informed consent forms. All procedures performed in this study involving human participants were in accordance with the Declaration of Helsinki (as revised in 2013).

\section{Cell culture}

Mouse $\beta$-cell line min6 (ATCC, HS-C200660, Manassas, MD, USA) was maintained in RPMI 1640 medium (Gibco, CA, USA) containing 10\% fetal bovine serum and $1 \%$ penicillin and streptomycin in $5 \% \mathrm{CO}_{2}$ at $37{ }^{\circ} \mathrm{C}$. To study the effects of KCNQ1OT1 and miRNA-223-3p on pyroptosis in $\mathrm{HCV}$ infection, $\mathrm{HCV}$-infected (transfected with pJFH1) human liver cancer cell line Huh7.5.1 cells 
(ATCC, XY-XB-3306, Manassas, MD, USA) were cultured, the $\mathrm{HCV}$ particles from the culture medium were purified, and the min 6 cells were incubated at a multiplicity of infection (MOI) of 1.0 for 72 hours. Huh7.5.1 cells were maintained in Dulbecco's modified Eagle medium (DMEM; Gibco, CA, USA) containing $10 \%$ fetal bovine serum, and $1 \%$ penicillin and streptomycin in $5 \% \mathrm{CO}_{2}$ at $37^{\circ} \mathrm{C}$.

\section{Cell transfection}

To construct the KCNQ1OT1 and NLRP3 overexpression vector. The full-length sequences of KCNQ1OT1 and NLRP3 were inserted into the pcDNA3.1 plasmid (pcDNA3.1, Invitrogen, Carlsbad, CA, USA) to obtain the KCNQ1OT1 and NLRP3 overexpression plasmid pcDNA3.1-KCNQ1OT1 and pcDNA3.1-NLRP3, and pcDNA3.1 plasmid as a negative control. Small interfering RNA targeting KCNQ1OT1 (si-KCNQ1OT1), miR223-3p mimic/inhibitor, and negative control (NC) mimics (GenePharma, Shanghai, China) were then synthesized. Cells were transfected with Lipofectamine 3000 (Invitrogen, Carlsbad, CA, USA) according to the manufacturer's protocol.

\section{Real-time quantitative PCR (RT-qPCR)}

Trizol reagent (Invitrogen, Carlsbad, CA, USA) was used to extract total RNA from cells, and a NanoDrop 1000 spectrophotometer (NanoDrop, DE, USA) and agarose gel electrophoresis (Invitrogen, Carlsbad, CA, USA) used to detect RNA concentration and quality. The RNA was reverse transcribed using a reverse transcription kit (Promega, Madison, WI, USA). The cDNA was amplificated and detected by the Real-Time PCR system (Bio-Rad, Hercules, CA, USA) using SYBR Premix Ex Taq II (Takara, Tokyo, Japan), and the $2^{-\Delta \Delta C T}$ method was used to calculate the relative expression levels of genes. GAPDH and U6 served as the internal control. These primers are listed in Table 1.

\section{Western blotting}

Total cell protein was separated by $12 \%$ sodium dodecylsulfate polyacrylamide gel electrophoresis (SDS-PAGE) and transferred to a polyvinylidene fluoride (PVDF) membrane, then $5 \%$ fat-free milk powder was added to block for $2 \mathrm{~h}$ at room temperature. The primary antibodies against NLRP3 (1:1,000; ab263899, Abcam, Cambridge, UK), caspase-1
(1:1,000; ab207802, Abcam), IL-1 $\beta$ (1:1,000; ab283818, Abcam), IL-18 (1:5,000; ab191860, Abcam), GSDMD (1:1,000; ab219800, Abcam), and GAPDH (1:1,000; ab9485, Abcam), were then incubated at $4{ }^{\circ} \mathrm{C}$ overnight. After rinsing with Tris-buffered saline with $0.1 \%$ Tween 20 (TBST), the PVDF membranes were incubated with secondary antibody (1:10,000, Invitrogen, Carlsbad, CA, USA) at room temperature for 2 hours. Enhanced chemiluminescence (ECL) blotting detection reagents (BioVision, Milpitas, CA, USA) developed chemiluminescence signals and blots were quantified with ImageJ software.

\section{Luciferase reporter assay}

To identify if KCNQ1OT1 and NLRP3 were direct targets of miR-223-3p, cells were transfected with recombinant plasmid containing the firefly luciferase gene. The miR-233$3 p$ mimic, miR-233-3p inhibitor and KCNQ1OT1-wild-type (WT)/mutant-type (MUT) recombinant plasmid were cotransfected into cells, as were the miR-233-3p and NLRP3WT/MUT recombinant plasmids. Luciferase activity was measured $48 \mathrm{~h}$ after transfection using the dual luciferase reporter assay system (Promega, Madison, WI, USA).

\section{TUNEL staining}

Cells or tissues were fixed with $4 \%$ paraformaldehyde at room temperature for $10 \mathrm{~min}$ and permeated with $0.5 \%$ Triton X-100 in phosphate-buffered saline (PBS) for $2 \mathrm{~min}$. Cells were then rinsed, and $50 \mu \mathrm{L}$ TUNEL detection solution (Roche, Basel, Switzerland) added, and the sample was incubated at $37^{\circ} \mathrm{C}$ for 1 hour in the dark. After washing three times with PBS, the slide was mounted with antifluorescence quenching mounting solution. Images were then taken by a fluorescence microscope. The nuclei were stained with DAPI and TUNEL staining was assessed.

\section{Enzyme-linked immunosorbent assay (ELISA)}

The serum obtained from the cell supernatant was preserved at $-80^{\circ} \mathrm{C}$ for subsequent analysis by an ELISA kit (MlBIO, Shanghai, China), and the levels of IL-1 $\beta$, IL-18, and insulin were determined following the instruction protocols.

\section{Determination of cell pyroptosis}

Cell pyroptosis was measured using the FAM fluorescent- 
Table 1 The primers of RT-qPCR used in this study

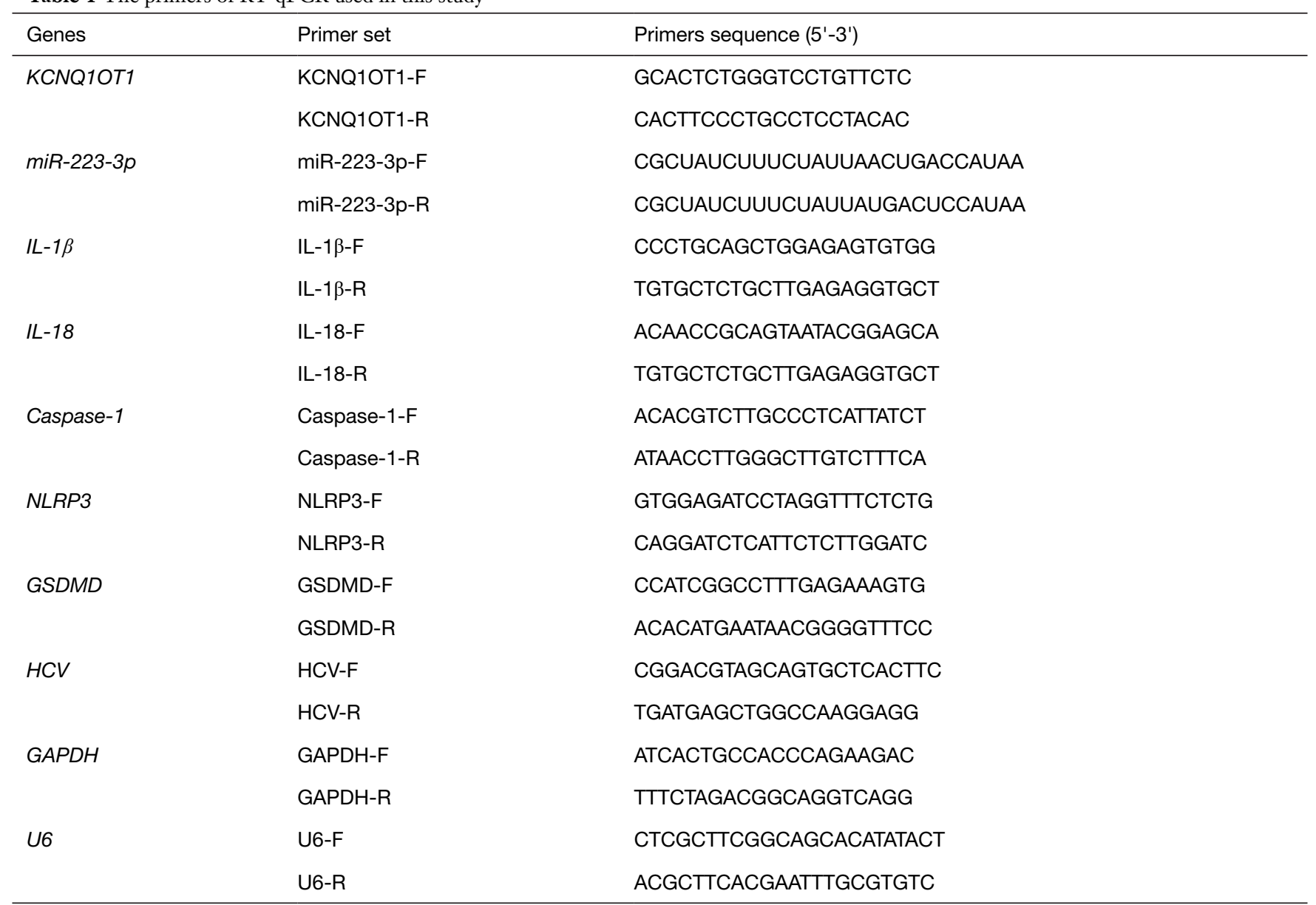

labelled inhibitor of caspase-1 assay (FLICA) and propidium iodide (PI) according to manufacturer's instruction (BioRad, Hercules, CA, USA). The fluorescent signal was detected using flow cytometry, and the percentage of pyroptosis cells was the percentage of active caspase-1-PI double-positive cells in total cells.

\section{Statistical analysis}

All experiments data were performed at least three times and values were expressed as mean \pm standard deviation (SD). SPSS software was used, and the data were analyzed using the Student's $t$-test and one-way analysis of variance (ANOVA). $\mathrm{P}<0.05$ was considered statistically significant.

\section{Results}

High expression of KCNQ1OT1 in T2DM patients and $\beta$-cell infected by $\mathrm{HCV}$

To explore the role of KCNQ1OT1 in HCV-infected T2DM, we detected the expression of KCNQ1OT1 in $\mathrm{HCV}$-infected T2DM patients and non-HCV-infected T2DM patients. Compared to patients with T2DM who were not infected with $\mathrm{HCV}$, those with T2DM who were infected had higher expression of KCNQ1OT1 (Figure 1A). In addition, we measured higher levels of pyroptosis-related proteins IL-1 $\beta$, IL-18, NLRP3, caspase-1, and GSMDD in T2DM patients infected with HCV (Figure 1B). We then evaluated the expression of KCNQ1OT1 and miR-223p 

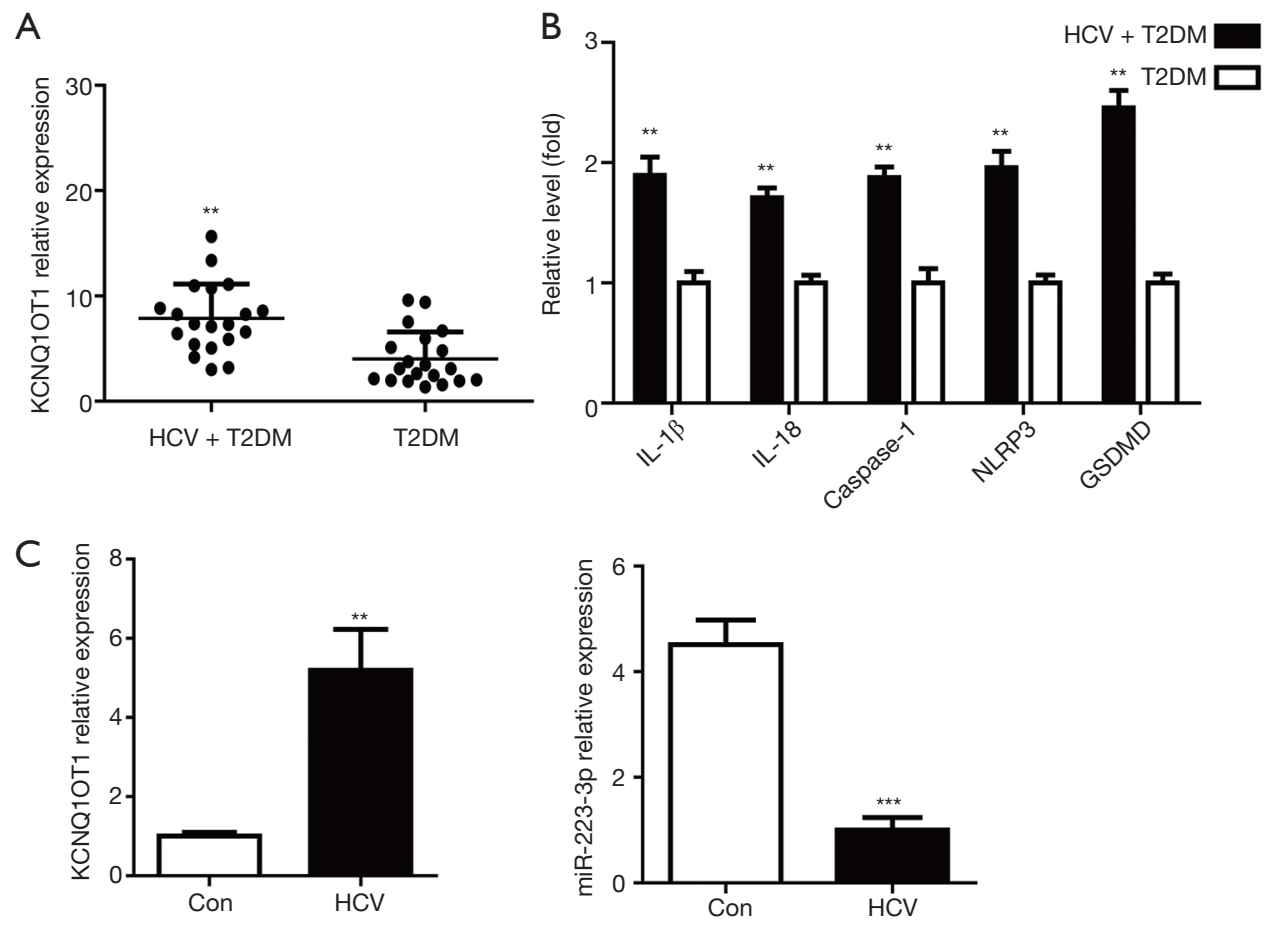

Figure 1 The expression of KCNQ1OT1 and apoptosis-related proteins increased under the induction of HCV. (A) RT-qPCR detected the expression of KCNQ1OT1. (B) ELISA detected the expression of IL-1 $\beta$, IL-18, NLRP3, caspase-1, and GSDMD. (C) The expression of KCNQ1OT1 and miR-223-3p revealed by RT-qPCR. (A,B) Compared with T2DM group, ** $\mathrm{P}<0.01$; (C) compared with Control (Con, min6 cells) group, ${ }^{* *} \mathrm{P}<0.01,{ }^{* * *} \mathrm{P}<0.001$. KCNQ1OT1, KCNQ1 overlapping transcript 1 ; HCV, hepatitis $\mathrm{C}$ virus; RT-qPCR, real-time quantitative PCR; T2DM, type 2 diabetes mellitus.

in HCV-infected min6 cells and found KCNQ1OT1 was significantly increased, and miR-223p was decreased after $\mathrm{HCV}$ infection (Figure 1C). Therefore, we determined that KCNQ1OT1 is highly expressed in HCV-infected T2DM patients and cells, and its increase may be related to pyroptosis.

\section{KCNQ1OT1 is involved in $\beta$-cell pyroptosis induced by HCV infection}

To clarify the relationship between KCNQ1OT1 and $\beta$-cell pyroptosis induced by $\mathrm{HCV}$ we constructed $\mathrm{HCV}$-infected min6 cells and detected the HCV copy number. The HCV copy number of the $\mathrm{HCV}$ group was higher than the control group (Figure $2 A$ ), indicating that our vitro model could be used. We then applied different small infectious RNAs (siRNA), which included siRNA NC (si-NC) and siRNAKCNQ1OT1 (si-K1/2/3), to knockdown KCNQ1OT1, and as si-K2 knockdown efficiency was the most significant (Figure 2B), we used it in subsequent studies. RT-qPCR and
Western blot were used to detect the mRNA and protein expression levels of IL-1 $\beta$, IL-18, NLRP3, caspase-1, and GSDMD, respectively. And the results showed that while HCV infection increased the expression of these proteins related to pyroptosis, knockdown of KCNQ1OT1 significantly reduced the expression (Figure 2C,2D). In ELISA analysis, insulin secretion decreased after $\mathrm{HCV}$ infection, and insulin levels were restored after HCVinfected cells were transfected with si- KCNQ1OT1 (Figure 2E). Moreover, we found that $\mathrm{HCV}$ promoted cell pyroptosis and transfection of si-KCNQ1OT1 alleviated pyroptosis in $\mathrm{HCV}$-infected cells (Figure 2F,2G). These results showed that KCNQ1OT1 regulated $\mathrm{HCV}$-infected pyroptosis of $\beta$-cells, and knockdown of KCNQ1OT1 inhibited cell pyroptosis in $\mathrm{HCV}$ infection.

\section{KCNQ1OT1 target regulates the miR-223-3p/NLRP3 axis}

To explain the mechanism of KCNQ1OT1 in HCV- 

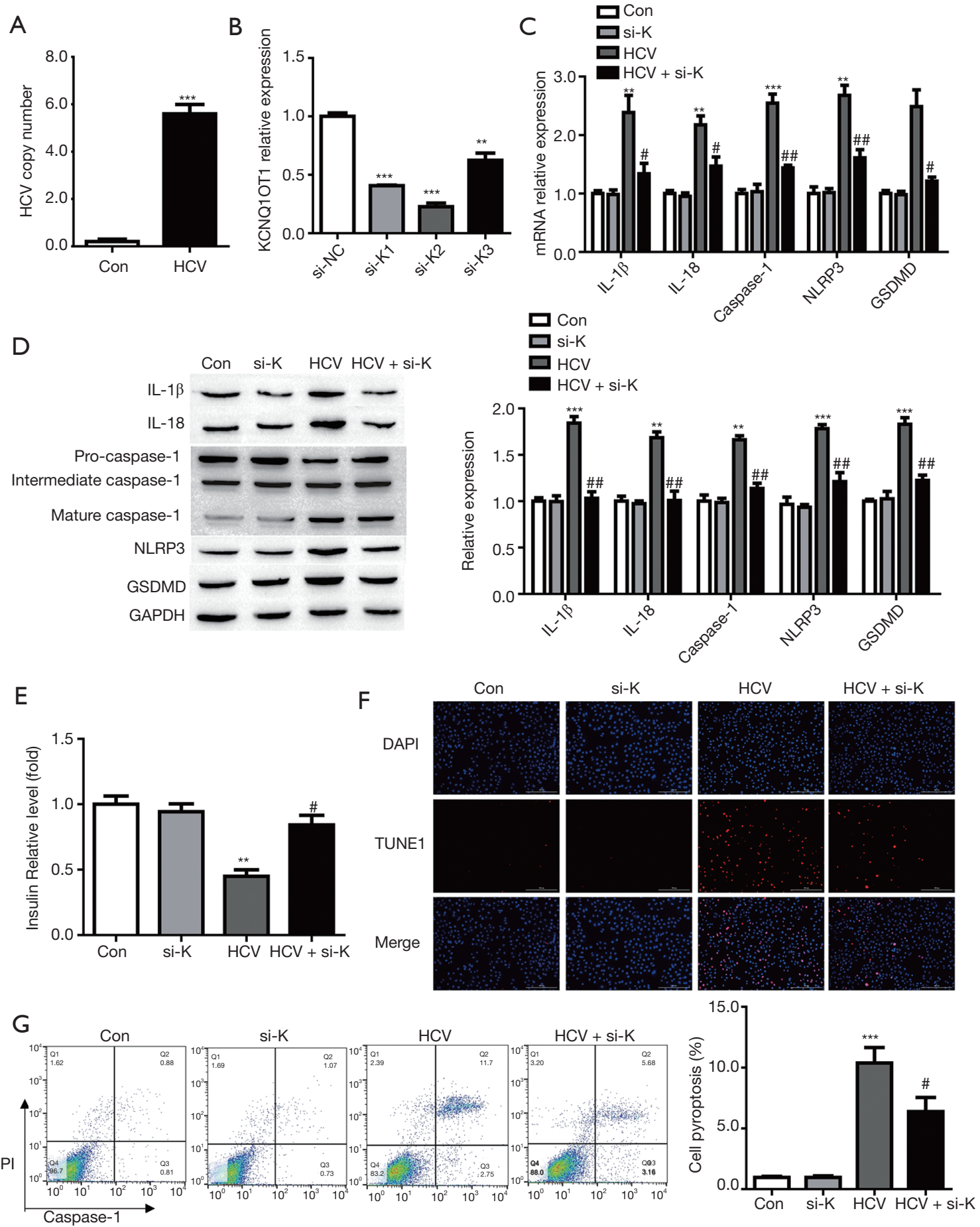

Figure 2 Knockdown of KCNQ1OT1 reduced $\beta$-cell pyroptosis induced by HCV. (A) RT-qPCR detected the HCV copy number. (B) RTqPCR detection of knockdown efficiency. (C) The mRNA expression of $I L-1 \beta, I L-18, N L R P 3$, caspase-1, and GSDMD measured by RTqPCR. (D) The protein expression of IL-1 $\beta$, IL-18, NLRP3, caspase-1, and GSDMD measured by Western blot. (E) ELISA to discover the level of insulin. (F) TUNEL determined cell death (scale: $200 \mu \mathrm{m}$ ). (G) Flow cytometry was used to analyze cell pyroptosis. Compared with Control (Con, min6 cells) group, ${ }^{* *} \mathrm{P}<0.01,{ }^{* * *} \mathrm{P}<0.001$; compared with $\mathrm{HCV}$ group, ${ }^{\#} \mathrm{P}<0.05,{ }^{, \#} \mathrm{P}<0.01$. KCNQ1OT1, KCNQ1 overlapping transcript 1; HCV, hepatitis C virus; RT-qPCR, real-time quantitative PCR; ELISA, enzyme-linked immunosorbent assay; si-K, small interfering RNA targeting KCNQ1OT1; PI, propidium iodide. 
A
KCNQ1OT1 WT
5' cuucccAUGUUCAC--AACUGACa 3'
| | | | | | || | | | |
miR-223-3p
3' accccaUA--AACUGUUUGACUGu 5'
KCNQ10T1 MUT

\section{5' cuucccAUGAACUG--UUGACUGa 3'}

B

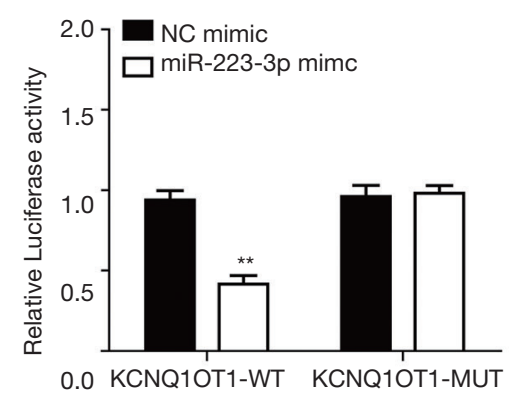

C

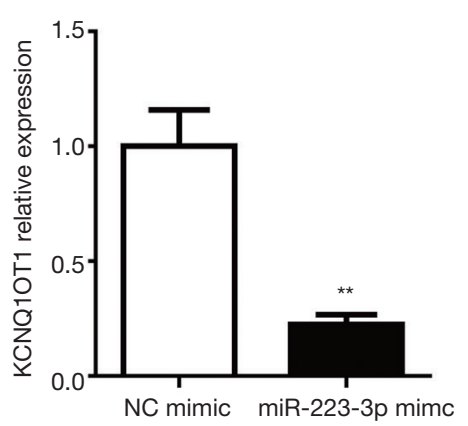

D

NLRP3 WT 5' CGCUAUCUUUCUAUUAACUGACC 3'

| | | | | | | | |

miR-223-3p 3' ACCCCAUAAACUGU--UUGACUGU 5'

NLRP3 MUT 5' CGCUAUCAAACUAUUUUGACUGC 3'

E

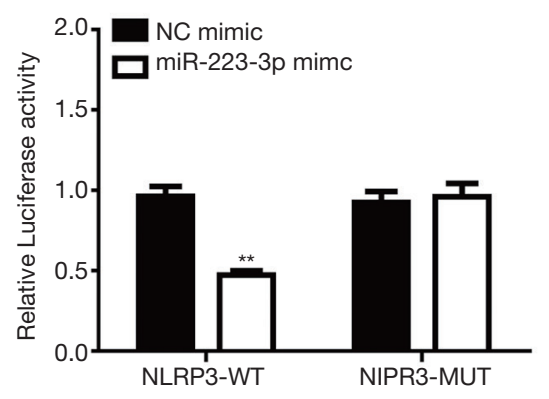

$\mathrm{F}$

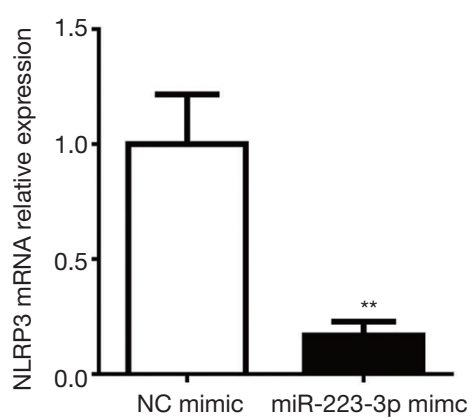

Figure 3 Targeting relationship of KCNQ1OT1, miR-223-3p, and NLRP3. (A) Sequence alignment of KCNQ1OT1, WT/MUT, and miR-223-3p. (B) Dual luciferase reporter gene assay to detect the targeting of KCNQ1OT1 and miR-223-3p. (C) The expression of KCNQ1OT1 presented by RT-qPCR. (D) Sequence alignment of NLRP3, WT/MUT, and miR-223-3p. (E) Dual luciferase reporter gene assay to detect the targeting of NLRP3 and miR-223-3p. (F) The expression of NLRP3 presented by RT-qPCR. Compared with NC group, ${ }^{* *} \mathrm{P}<0.01$. KCNQ1OT1, KCNQ1 overlapping transcript 1; WT, wild-type; MUT, mutant-type; RT-qPCR, real-time quantitative PCR; $\mathrm{NC}$, negative control.

induced $\beta$-cell pyroptosis, we predicted the miRNA regulated by KCNQ1OT1 through the bioinformatics website "Starbase", and discovered miR-223-3p (Figure 3A). Dual luciferase reporter assay was performed to verify the interaction between KCNQ1OT1 and miR-223-3p. The
KCNQ1OT1 3'-UTR WT fragments (KCNQ1OT1 WT) or MUT fragments (KCNQ1OT1 MUT) recombinant plasmids were co-transfected with the NC mimic or miR223-3p mimic into cells, and the results showed that the luciferase activity of co-transfected KCNQ1OT1-WT and 
miR-223-3p mimics was significantly reduced (Figure 3B). Furthermore, the expression level of KCNQ1OT1 was evaluated after transfection with the miR-223-3p mimic and was found to have decreased (Figure 3C). Similarly, we found that the target gene of miR-223-3p was NLRP3, which is a key protein (Figure 3D). Compared with cotransfection with NLRP3-Wt and NC mimic, the luciferase activity of NLRP3-Wt and the miR-223-3p mimic decreased significantly (Figure $3 E$ ), and the expression of NLRP3 was down-regulated after transfection with the miR-223-3p mimic (Figure 3F). Therefore, we speculate that KCNQ1OT1 participates in the regulation of $\beta$-cell pyroptosis with $\mathrm{HCV}$-induced through the miR-223-3p/ NLRP3 axis.

\section{KCNQ1OT1 regulates pyroptosis of $\beta$-cell infected by $\mathrm{HCV}$ through miRNA-223-3p}

We further confirmed the role of miR-223-3p in KCNQ1OT1's regulation of HCV-infected $\beta$-cell pyroptosis. After $\mathrm{HCV}$ induced min6 cells, overexpression of KCNQ1OT1 or miR-223-3p and KCNQ1OT1 at the same time, and transfected the empty vector (pcDNA3.1) as a negative control. Overexpression of miRNA-223-3p reduced the mRNA and protein expression levels of IL-1 $\beta$, IL-18, NLRP3, caspase-1, and GSDMD, while KCNQ1OT1 restored this reduction (Figure $4 A, 4 B$ ). In addition, we investigated insulin secretion by ELISA assay, and the results showed that overexpression of miR-223-3p increased insulin secretion, and overexpression of $\mathrm{miR}-223-3 \mathrm{p}$ and KCNQ1OT1 decreased insulin secretion (Figure $4 C$ ). In the flow cytometry analysis, we demonstrated that miRNA-223-3p played an important role in cell pyroptosis, overexpression of miRNA-223-3p obviously decreased cell pyroptosis, and the change could be reversed though the overexpression of KCNQ1OT1 (Figure 4D). These results indicated that KCNQ1OT1 regulated $\beta$-cell pyroptosis under $\mathrm{HCV}$-infection via targeting miRNA-223-3p.

\section{HCV induces $\beta$-cell pyroptosis by regulating KCNQ1OT1/ miRNA-223-3p/NLRP3}

Furthermore, we investigated the interplay among KCNQ1OT1, miR-223-3p, and NLRP3 in $\beta$-cell pyroptosis which were HCV-infected. The result of RTqPCR showed that si-KCNQ1OT1 (si-K) significantly decreased the mRNA expressions of $I L-1 \beta, I L-18, N L R P 3$, caspase-1, and GSDMD, compared with the HCV-infected group, while co-transfection with miR-223-3p inhibitor (inh) or overexpression of NLRP3 increased the mRNA expression of these proteins (Figure $5 A$ ). Similarly, knockdown of KCNQ1OT1 increased insulin secretion, and inhibition of miR-223-3p or overexpression of NLRP3 reversed this effect (Figure 5B). Further, Western blot demonstrated the expression of IL-1 $\beta$, IL-18, NLRP3, caspase-1, and GSDMD, and showed their expression decreased in cells which were transfected with si-K. After inhibiting miR-223-3p or overexpressing NLRP3, the expression of IL-1 $\beta$, IL-18, NLRP3, caspase-1, and GSDMD increased, which was consistent with the mRNA results (Figure 5C). Measurement of cell pyroptosis by flow cytometry proved that knockdown of KCNQ1OT1 reduced pyroptosis, while inhibiting miR-223-3p or overexpression of NLRP3 inhibited the cell pyroptosis induced by KCNQ1OT1 (Figure 5D). Consequently, we concluded that the KCNQ1OT1/miR-223-3p/NLRP3 axis regulates $\beta$-cell pyroptosis infected by $\mathrm{HCV}$.

\section{Discussion}

T2DM is caused by the joint participation and interaction of multiple risk factors, and its incidence has continued to rise globally. The pathogenesis mainly involves pancreatic $\beta$-cell dysfunction and insulin resistance, while inflammation plays a mediator role in its pathogenesis. Studies (32-34) have shown that HCV infection is significantly related to $\mathrm{T} 2 \mathrm{DM}$, and insulin resistance and T2DM are more common in patients this infection. However, the mechanism by which $\mathrm{HCV}$ infection is related to the occurrence and development of T2DM is still unclear. One possible mechanism may involve damage to $\beta$-cells. Pyroptosis is a newly discovered inflammatory form of programmed cell death. When the NLRP3 inflammasome is activated, caspase- 1 is activated, which then mediates the activation of downstream inflammatory factors (IL-1 $\beta$ and IL-18) and the lysis of GSDMD to trigger the production of pyroptosis (35-37). Pyroptosis is not only related to diabetes and its complications but is closely related to $\mathrm{HCV}$ virus infection. Therefore, we studied the mechanism of HCV infectioninduced $\beta$-cell pyroptosis leading to T2DM.

LncRNA exerts biological effects through a variety of mechanisms. LncRNA KCNQ1OT1 is abnormally expressed in a variety of diseases and plays different roles among them (38-40). Our study found that KCNQ1OT1 was highly expressed in $\mathrm{HCV}$-infected T2DM patients and cells, and cell experiments proved that KCNQ1OT1 

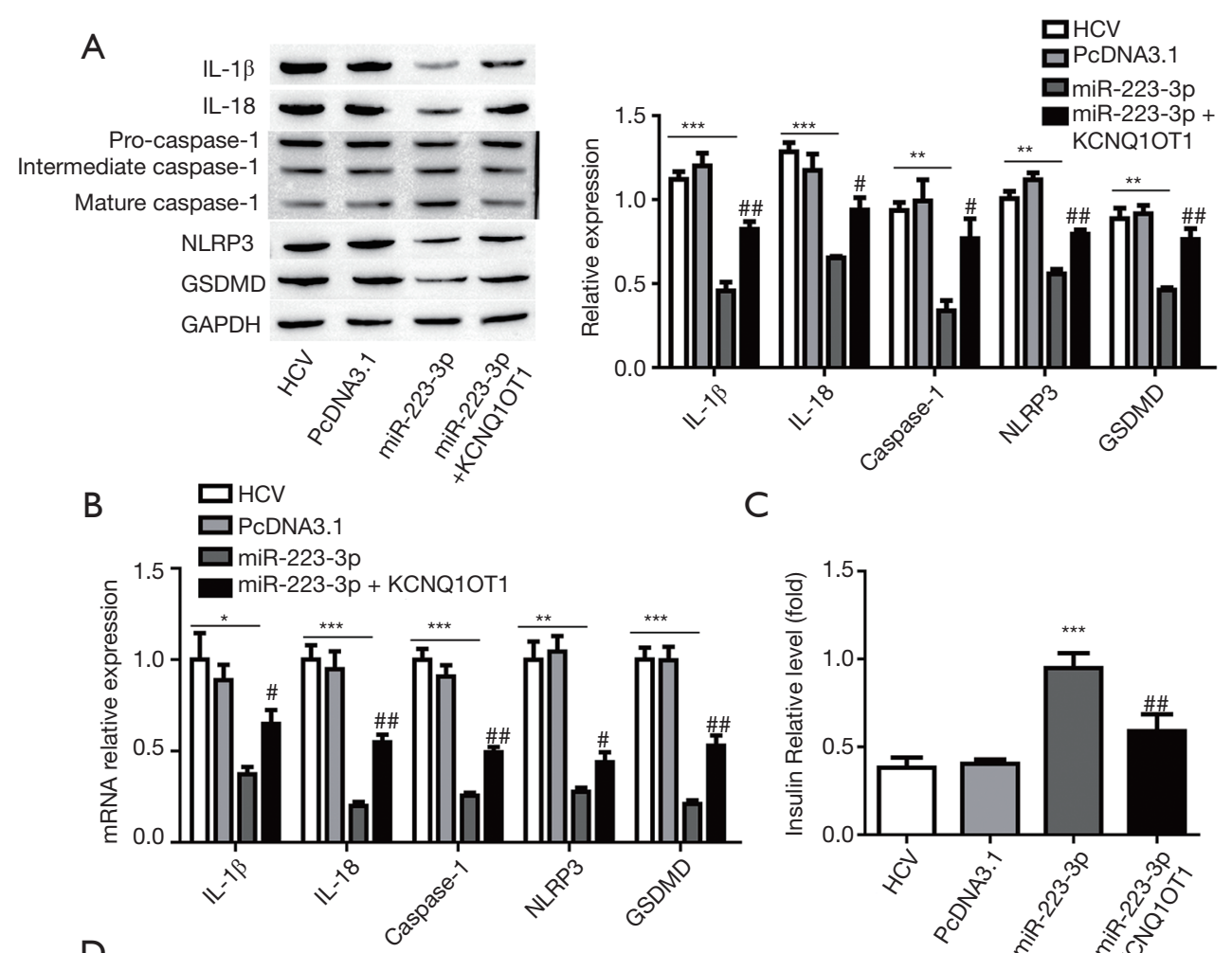

C
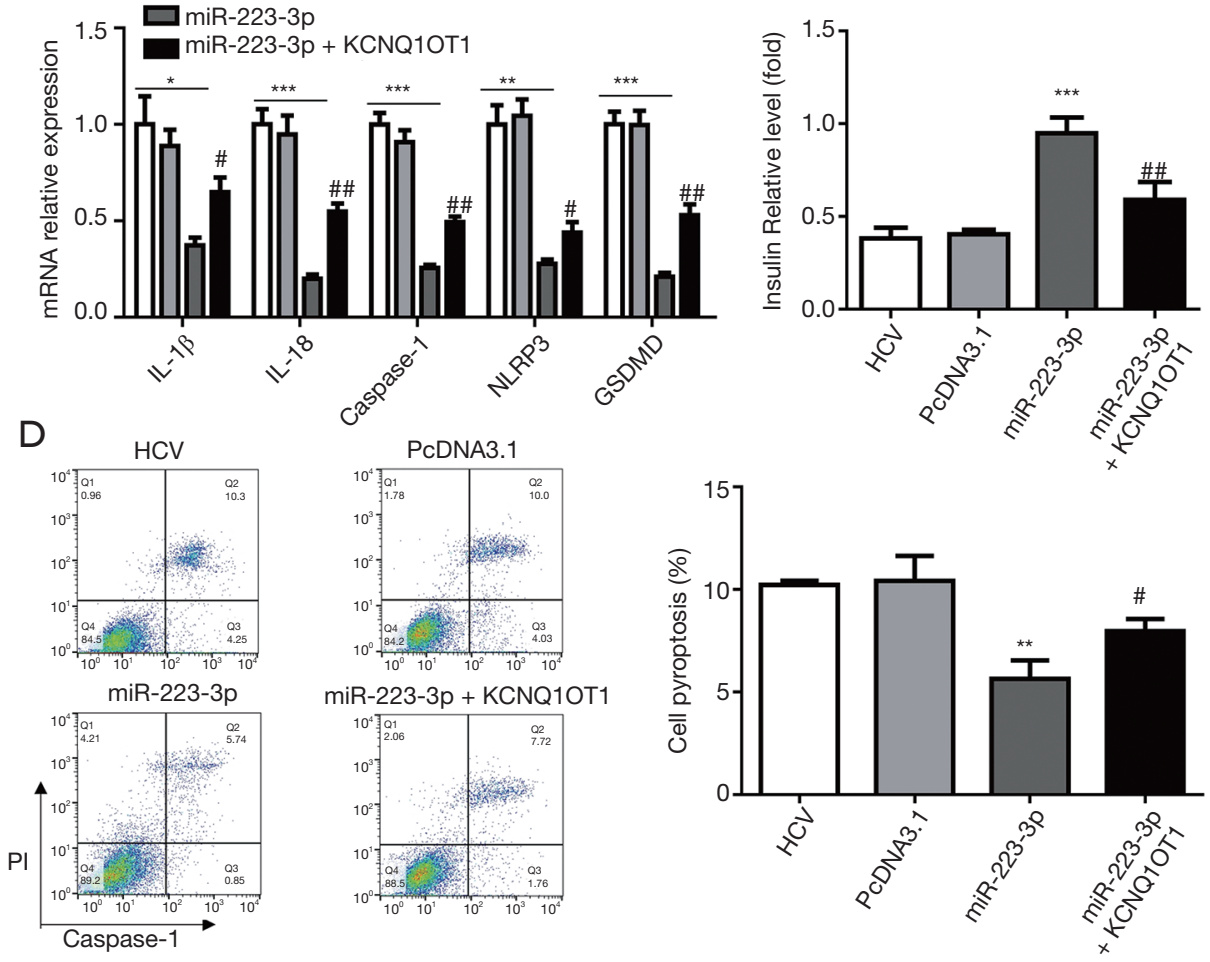

Figure 4 KCNQ1OT1 regulates $\beta$-cell pyroptosis under HCV-infected via targeting miRNA-223-3p. (A) The protein expression of IL1 $\beta$, IL-18, NLRP3, caspase-1, and GSDMD presented by Western blot. (B) RT-qPCR detection of the mRNA expression of $I L-1 \beta, I L-$ 18, NLRP3, caspase-1, and GSDMD. (C) ELISA to measure the level of insulin. (D) Flow cytometry was used to analyze cell pyroptosis. All groups were infected with HCV. Compared with HCV group, ${ }^{*} \mathrm{P}<0.05$, ${ }^{* *} \mathrm{P}<0.01$, ${ }^{* * *} \mathrm{P}<0.001$; compared with miR-223-3p group, ${ }^{*} \mathrm{P}<0.05$, ${ }^{\# \#} \mathrm{P}<0.01$. KCNQ1OT1, KCNQ1 overlapping transcript 1; HCV, hepatitis C virus; RT-qPCR, real-time quantitative PCR; ELISA, enzymelinked immunosorbent assay; PI, propidium iodide.

silence after $\mathrm{HCV}$ infection reduced $\beta$-cell pyroptosis, indicating that KCNQ1OT1 plays a considerable role in T2DM induced by HCV infection. LncRNA regulates downstream target genes by binding miRNA and is associated with pyroptosis. For example, lncRNA MALAT1/
miR-23c/ELAVL1 regulated renal tubular epithelial cell pyroptosis in diabetic nephropathy (41), lncRNA MALAT1/ miR-30c/NLRP3 promoted renal tubular epithelial cell pyroptosis induced by high glucose (HG) (42), and lncRNA KCNQ1OT1/miR-214-3p/NLRP3 regulated the pyroptosis 


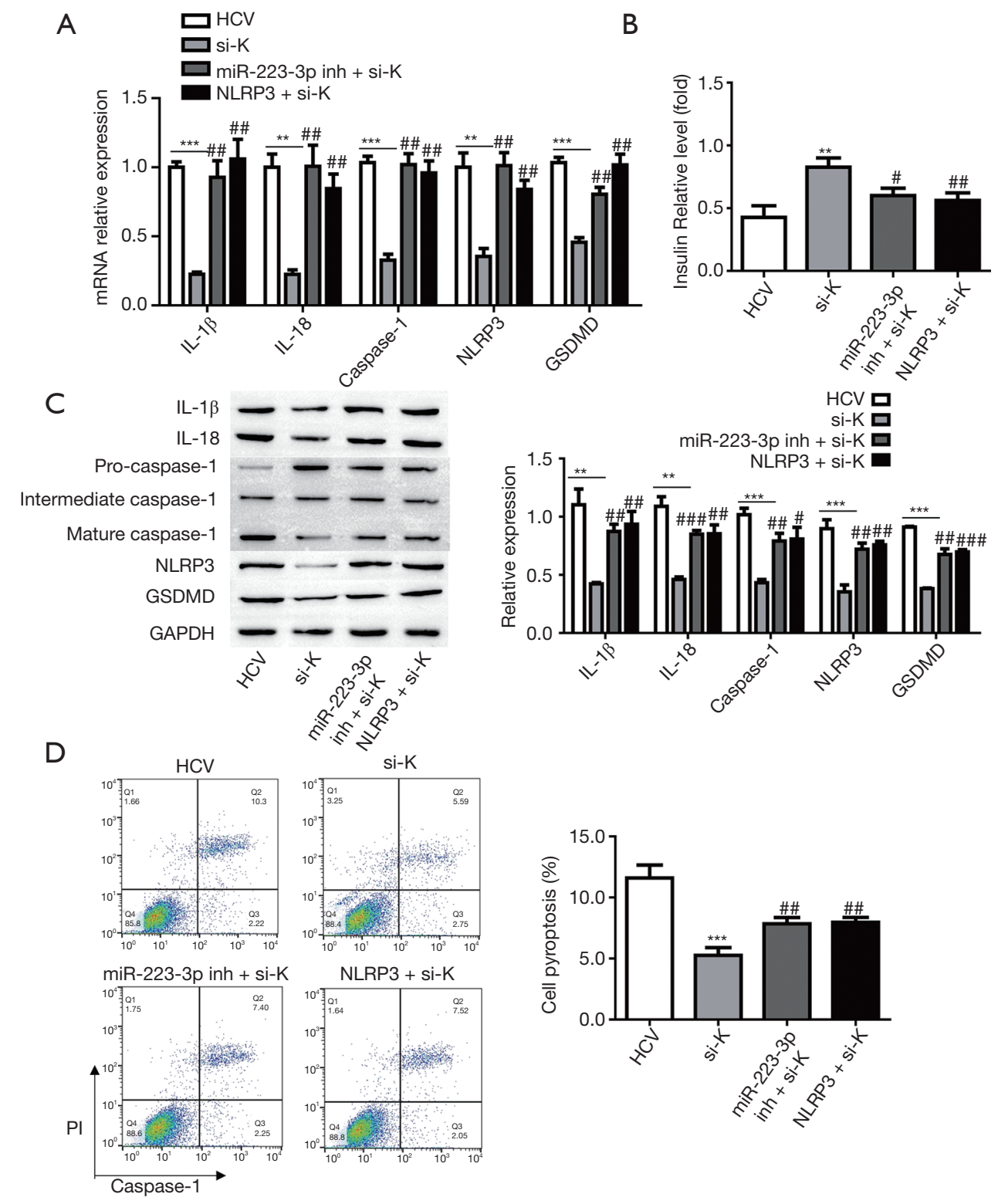

Figure $5 \mathrm{KCNQ} 1 \mathrm{OT} 1 / \mathrm{miR}-223-3 \mathrm{p} / \mathrm{NLRP} 3$ axis participates in HCV-induced $\beta$-cell pyroptosis. (A) RT-qPCR detection of the mRNA expression of $I L-1 \beta, I L-18, N L R P 3$, caspase-1, and GSDMD. (B) ELISA to measure the level of insulin. (C) The protein expression of IL1 $\beta$, IL-18, NLRP3, caspase-1, and GSDMD were presented by Western blot. (D) Flow cytometry was used to analyze cell pyroptosis. All groups were infected with HCV. Compared with $\mathrm{HCV}$ group, ${ }^{* *} \mathrm{P}<0.01,{ }^{* * *} \mathrm{P}<0.001$; compared with si-K group, ${ }^{*} \mathrm{P}<0.05,{ }^{*} \mathrm{P}<0.01$,

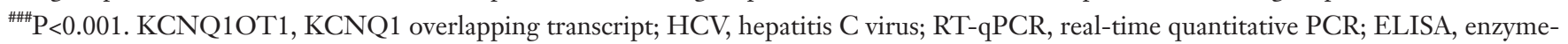
linked immunosorbent assay; si-K, small interfering RNA targeting KCNQ1OT1; PI, propidium iodide; inh, inhibitor.

of cardiomyocytes in diabetic cardiomyopathy (43).

However, the molecular mechanism of lncRNA/ miRNA regulating $\beta$-cell pyroptosis in T2DM induced by $\mathrm{HCV}$ infection has not been reported. We predicted the potential miRNA of KCNQ1OT1 through "Starbase",
miR-223-3p and KCNQ1OT1 have binding sites. miR$223-3 p$ could be used as a potential biomarker in diabetes and its complications (44), and, importantly, was closely related to $\mathrm{HCV}$-infected liver cirrhosis and HCC (45). Our data indicated that miR-223-3p is lowly expressed 


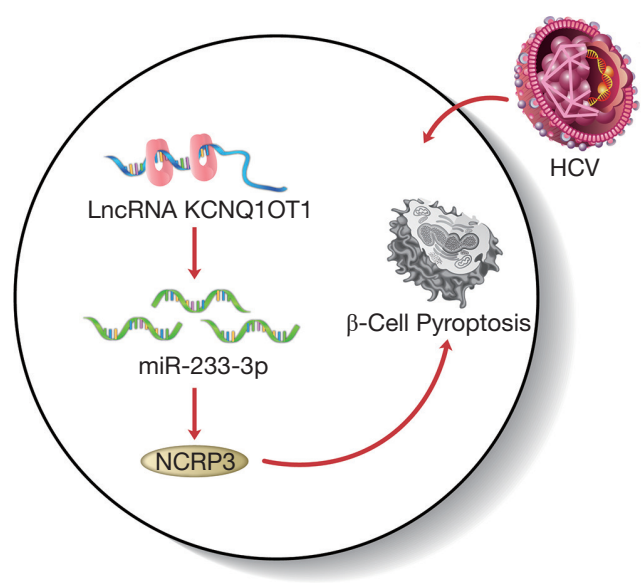

Figure 6 Schematic diagram of the mechanism of the KCNQ1OT1/miR-223-3p/NLRP3 axis involved in HCV-induced $\beta$ cell pyroptosis. KCNQ1OT1, KCNQ1 overlapping transcript 1; $\mathrm{HCV}$, hepatitis $\mathrm{C}$ virus.

in $\mathrm{HCV}$-infected $\beta$-cells, and its overexpression inhibited cell pyroptosis, while KCNQ1OT1 abolished the inhibitory effect of miR-223-3p, which further proved that KCNQ1OT1 regulates $\mathrm{HCV}$-infected cell pyroptosis through miR-223-3p. The pyroptosis related protein NLRP3 is a target gene of miR-223-3p. In this study, we explored NLRP3 under the induction of HCV, and found KCNQ1OT1/miR-223-3p targeted and regulated NLRP3, which affected the expression of IL-1 $\beta$, IL-18, caspase-1, and GSDMD, thereby regulating $\beta$-cell pyroptosis. Taken together, these results suggest KCNQ1OT1 acts as a sponge of miR-223-3p to affect the expression of NLRP3 and participates in the pathogenesis of T2DM induced by HCV infection by regulating the apoptosis of $\beta$ cells (Figure 6).

In summary, our study proved that KCNQ1OT1 promotes the $\mathrm{HCV}$-infected pyroptosis of $\beta$-cells by regulating the miRNA-223-3p/NLRP3 axis, effecting the production of insulin and accelerating the occurrence and development of T2DM. Regulating KCNQ1OT1 and its target genes will help to better understand the pathogenesis of T2DM induced by HCV infection and provide new theoretical foundations and therapeutic targets.

\section{Acknowledgments}

Funding: This study was supported by the Joint Special Fund Project of Yunnan Provincial Science and Technology Department and Kunming Medical University for Applied Basic Research, grant number: 2018FE001(-127), the
Regional Science Foundation Project of National Natural Science Foundation of China, grant number: 81960150, and the Yunnan Provincial Young and Middle-aged Academic and Technical Leaders Reserve Talents Program, grant number: 202105AC160028.

\section{Footnote}

Reporting Checklist: The authors have completed the MDAR reporting checklist. Available at https://dx.doi. org/10.21037/atm-21-3862

Data Sharing Statement: Available at https://dx.doi. org/10.21037/atm-21-3862

Conflicts of Interest: All authors have completed the ICMJE uniform disclosure form (available at https://dx.doi. org/10.21037/atm-21-3862). The authors have no conflicts of interest to declare.

Ethical Statement: The authors are accountable for all aspects of the work in ensuring that questions related to the accuracy or integrity of any part of the work are appropriately investigated and resolved. The study received approval from the ethics committee of the First People's Hospital of Yunnan Province (KHLL2020-KY059), and all participants signed informed consent forms. All procedures performed in this study involving human participants were in accordance with the Declaration of Helsinki (as revised in 2013).

Open Access Statement: This is an Open Access article distributed in accordance with the Creative Commons Attribution-NonCommercial-NoDerivs 4.0 International License (CC BY-NC-ND 4.0), which permits the noncommercial replication and distribution of the article with the strict proviso that no changes or edits are made and the original work is properly cited (including links to both the formal publication through the relevant DOI and the license). See: https://creativecommons.org/licenses/by-nc-nd/4.0/.

\section{References}

1. Saisho Y. $\beta$-cell dysfunction: its critical role in prevention and management of type 2 diabetes. World J Diabetes 2015;6:109-24.

2. Li L, Wang C, Yang H, et al. Metabolomics reveal mitochondrial and fatty acid metabolism disorders that 
contribute to the development of DKD in T2DM patients. Mol Biosyst 2017;13:2392-400.

3. Halvorsen B, Santilli F, Scholz H, et al. LIGHT/ TNFSF14 is increased in patients with type 2 diabetes mellitus and promotes islet cell dysfunction and endothelial cell inflammation in vitro. Diabetologia 2016;59:2134-44.

4. Chen Y, Ji H, Shao J, et al. Different hepatitis C virus infection statuses show a significant risk of developing type 2 diabetes mellitus: a network meta-analysis. Dig Dis Sci 2020;65:1940-50.

5. Adinolfi LE, Jacobson I, Bondin M, et al. Expert opinion on managing chronic $\mathrm{HCV}$ infection in patients with type 2 diabetes mellitus. Antivir Ther 2018;23:11-21.

6. Shlomai A, Rechtman MM, Burdelova EO, et al. The metabolic regulator PGC-1 $\alpha$ links hepatitis C virus infection to hepatic insulin resistance. $\mathrm{J}$ Hepatol 2012;57:867-73.

7. Shoji I, Deng L, Matsui C, et al. Hepatitis C virus-induced glucose metabolic disorder. Uirusu 2015;65:263-8.

8. Huang JF, Yu ML, Dai CY, et al. Glucose abnormalities in hepatitis $\mathrm{C}$ virus infection. Kaohsiung J Med Sci 2013;29:61-8.

9. Antonelli A, Ferrari SM, Giuggioli D, et al. Hepatitis C virus infection and type 1 and type 2 diabetes mellitus. World J Diabetes 2014;5:586-600.

10. Fleisher TA. Apoptosis. Ann Allergy Asthma Immunol 1997;78:245-9; quiz 249-50.

11. Bergsbaken T, Fink SL, Cookson BT. Pyroptosis: host cell death and inflammation. Nat Rev Microbiol 2009; 7:99-109.

12. Man SM, Karki R, Kanneganti TD. Molecular mechanisms and functions of pyroptosis, inflammatory caspases and inflammasomes in infectious diseases. Immunol Rev 2017;277:61-75.

13. Rojas J, Bermudez V, Palmar J, et al. Pancreatic beta cell death: novel potential mechanisms in diabetes therapy. J Diabetes Res 2018;2018:9601801.

14. Pei P, Yao X, Jiang L, et al. Inorganic arsenic induces pyroptosis and pancreatic $\beta$ cells dysfunction through stimulating the IRE $1 \alpha / \mathrm{TNF}-\alpha$ pathway and protective effect of taurine. Food Chem Toxicol 2019;125:392-402.

15. Eizirik DL, Millard I. Chronicle of a death foretold: endoplasmic reticulum stress and beta cell apoptosis in diabetes. Med Sci (Paris) 2014;30:496-9.

16. Kofahi HM, Taylor NG, Hirasawa K, et al. Hepatitis C virus infection of cultured human hepatoma cells causes apoptosis and pyroptosis in both infected and bystander cells. Sci Rep 2016;6:37433.
17. Negro F, Alaei M. Hepatitis C virus and type 2 diabetes. World J Gastroenterol 2009;15:1537-47.

18. Beermann J, Piccoli MT, Viereck J, et al. Non-coding RNAs in development and disease: background, mechanisms, and therapeutic approaches. Physiol Rev 2016;96:1297-325.

19. Goff LA, Rinn JL. Linking RNA biology to lncRNAs. Genome Res 2015;25:1456-65.

20. Xue M, Zhuo Y, Shan B. MicroRNAs, long noncoding RNAs, and their functions in human disease. Methods Mol Biol 2017;1617:1-25.

21. Zhang C, Yang X, Qi Q, et al. lncRNA-HEIH in serum and exosomes as a potential biomarker in the HCV-related hepatocellular carcinoma. Cancer Biomark 2018;21:651-9.

22. Liu X, Duan X, Holmes JA, et al. A long noncoding RNA regulates hepatitis $\mathrm{C}$ virus infection through interferon alpha-inducible protein 6. Hepatology 2019;69:1004-19.

23. Jin F, Wang N, Zhu Y, et al. Downregulation of Long Noncoding RNA Gas5 Affects Cell Cycle and Insulin Secretion in Mouse Pancreatic $\beta$ Cells. Cell Physiol Biochem 2017;43:2062-73.

24. You L, Wang N, Yin D, et al. Downregulation of long noncoding RNA Meg3 affects insulin synthesis and secretion in mouse pancreatic beta cells. J Cell Physiol 2016;231:852-62.

25. Yin DD, Zhang EB, You LH, et al. Downregulation of lncRNA TUG1 affects apoptosis and insulin secretion in mouse pancreatic $\beta$ cells. Cell Physiol Biochem 2015;35:1892-904.

26. Yang F, Qin Y, Wang Y, et al. LncRNA KCNQ1OT1 mediates pyroptosis in diabetic cardiomyopathy. Cell Physiol Biochem 2018;50:1230-44.

27. Chen L, Heikkinen L, Wang C, et al. Trends in the development of miRNA bioinformatics tools. Brief Bioinform 2019;20:1836-52.

28. Krol J, Loedige I, Filipowicz W. The widespread regulation of microRNA biogenesis, function and decay. Nat Rev Genet 2010;11:597-610.

29. Zhang Y, Liu X, Bai X, et al. Melatonin prevents endothelial cell pyroptosis via regulation of long noncoding RNA MEG3/miR-223/NLRP3 axis. J Pineal Res 2018. doi: 10.1111/jpi.12449.

30. Parrizas M, Mundet X, Castaño C, et al. miR-10b and miR-223-3p in serum microvesicles signal progression from prediabetes to type 2 diabetes. J Endocrinol Invest 2020;43:451-9.

31. Oksuz Z, Serin MS, Kaplan E, et al. Serum microRNAs; miR-30c-5p, miR-223-3p, miR-302c-3p and miR-17-5p 
could be used as novel non-invasive biomarkers for HCVpositive cirrhosis and hepatocellular carcinoma. Mol Biol Rep 2015;42:713-20.

32. Fabiani S, Fallahi P, Ferrari SM, et al. Hepatitis C virus infection and development of type 2 diabetes mellitus: Systematic review and meta-analysis of the literature. Rev Endocr Metab Disord 2018;19:405-20.

33. Shiffman ML, Gunn NT. Impact of hepatitis $\mathrm{C}$ virus therapy on metabolism and public health. Liver Int 2017;37 Suppl 1:13-8.

34. Guo X, Jin M, Yang M, et al. Type 2 diabetes mellitus and the risk of hepatitis $\mathrm{C}$ virus infection: a systematic review. Sci Rep 2013;3:2981.

35. Xue Y, Enosi Tuipulotu D, Tan WH, et al. Emerging activators and regulators of inflammasomes and pyroptosis. Trends Immunol 2019;40:1035-52.

36. Kesavardhana S, Kanneganti TD. Mechanisms governing inflammasome activation, assembly and pyroptosis induction. Int Immunol 2017;29:201-10.

37. Tsuchiya K. Inflammasome-associated cell death: pyroptosis, apoptosis, and physiological implications. Microbiol Immunol 2020;64:252-69.

38. Dong Z, Yang P, Qiu X, et al. KCNQ1OT1 facilitates progression of non-small-cell lung carcinoma via modulating miRNA-27b-3p/HSP90AA1 axis. J Cell Physiol 2019;234:11304-14.

39. Li X, Dai Y, Yan S, et al. Down-regulation of lncRNA KCNQ1OT1 protects against myocardial ischemia/

Cite this article as: Niu B, Yao L, Zhang Y, Xia X, Su H. LncRNA KCNQ1OT1 promoted hepatitis C virus-induced pyroptosis of $\beta$-cell through mediating the miR-223-3p/NLRP3 axis. Ann Transl Med 2021;9(17):1387. doi: 10.21037/atm-213862 reperfusion injury following acute myocardial infarction. Biochem Biophys Res Commun 2017;491:1026-33.

40. Zhang S, Ma H, Zhang D, et al. LncRNA KCNQ1OT1 regulates proliferation and cisplatin resistance in tongue cancer via miR-211-5p mediated Ezrin/Fak/Src signaling. Cell Death Dis 2018;9:742.

41. Li X, Zeng L, Cao C, et al. Long noncoding RNA MALAT1 regulates renal tubular epithelial pyroptosis by modulated miR-23c targeting of ELAVL1 in diabetic nephropathy. Exp Cell Res 2017;350:327-35.

42. Liu C, Zhuo H, Ye MY, et al. LncRNA MALAT1 promoted high glucose-induced pyroptosis of renal tubular epithelial cell by sponging miR-30c targeting for NLRP3. Kaohsiung J Med Sci 2020;36:682-91.

43. Yang F, Qin Y, Lv J, et al. Silencing long non-coding RNA Kcnq1ot1 alleviates pyroptosis and fibrosis in diabetic cardiomyopathy. Cell Death Dis 2018;9:1000.

44. Shao J, Fan G, Yin X, et al. A novel transthyretin/ STAT4/miR-223-3p/FBXW7 signaling pathway affects neovascularization in diabetic retinopathy. Mol Cell Endocrinol 2019;498:110541.

45. Ghosh S, Bhowmik S, Majumdar S, et al. The exosome encapsulated microRNAs as circulating diagnostic marker for hepatocellular carcinoma with low alpha-fetoprotein. Int J Cancer 2020;147:2934-47.

(English Language Editor: B. Draper) 